Annals of Pure and Applied Mathematics

Vol. 15, No. 1, 2017, 1-11

ISSN: 2279-087X (P), 2279-0888(online)

Published on 11 December 2017

Annals of

www.researchmathsci.org

DOI: http://dx.doi.org/10.22457/apam.v15n1al

Pure and Applied

Mathematics

\title{
Bounds of Location-2-Domination Number for Products of Graphs
}

\author{
G.Rajasekar $^{1}$ and A.Venkatesan ${ }^{2}$ \\ ${ }^{1}$ Department of Mathematics, Jawahar Science College, Neyveli. \\ Tamilnadu, India. Email: grsmaths@ gmail.com \\ ${ }^{2}$ Department of Mathematics, St. Joseph's College of Arts and Science College \\ (Autonomous) Cuddalore. Tamilnadu, India. \\ Email: suresh11venkat@ gmail.com
}

Received 1 November 2017; accepted 4 December 2017

Abstract. In this paper Location-2-Domination set and their properties are being studied. A subset $S \subseteq V$ is Location-2-Dominating set of $\mathrm{G}$ if $\mathrm{S}$ is 2-Dominating set of $\mathrm{G}$ and for any two vertices $u, v \in V-S$ such that $N(u) \cap S \neq N(v) \cap S$, its denoted by $R_{2}^{D}(G)$. Based on this definition the bounds of the Location-2-domination number for direct product, Cartesian product and semi-strong product of graphs namely $P_{n} \square C_{m}$, $C_{n} \square S_{m}, P_{n} \times W_{m}, C_{n} \times S_{m} \mathrm{P}_{\mathrm{n}} \bowtie \mathrm{P}_{\mathrm{m}}, \mathrm{C}_{\mathrm{n}} \bowtie \mathrm{P}_{\mathrm{m}}, \mathrm{C}_{\mathrm{n}} \bowtie \mathrm{C}_{\mathrm{m}}$ have been found.

Keywords: 2-Domination, Location Domination, Product of Graphs

AMS Mathematics Subject Classification (2010): 05 C69

\section{Introduction}

Throughout this paper let us follow the terminology and notation of Harary [11]. Cockayne and Hedetniemi [7] introduce the concept dominating set. A subset S of vertices from $\mathrm{V}$ is called a dominating set for $G$ if every vertex of $G$ is either a member of $\mathrm{S}$ or adjacent to a member of S. A dominating set of $G$ is called a minimum dominating set if $G$ has no dominating set of smaller cardinality. The cardinality of minimum dominating set of $G$ is called the dominating number for $G$ and it is denoted by $\gamma(G)[6]$.

Harary and Haynes [5] introduced the concepts of double domination in graphs. A dominating set $\mathrm{S}$ of $G$ is called double dominating set if every vertex in V-S is adjacent to at least two vertices in $\mathrm{S}$. Given a dominating set $\mathrm{S}$ for graph $G$, for each u in $\mathrm{V}-\mathrm{S}$ let $\mathrm{S}(\mathrm{u})$ denote the set of vertices in $\mathrm{S}$ which are adjacent to $\mathrm{u}$. The set $\mathrm{S}$ is called locating dominating set, if for any two vertices $u$ and $w$ in $\mathrm{V}-\mathrm{S}$ one has $\mathrm{S}(\mathrm{u})$ not equal to $\mathrm{S}(\mathrm{w})$ and the minimum cardinality of Location Domination set is denoted by $\operatorname{RD}(\mathrm{G})$ [7]. The Cartesian product $G \square H$ of graphs $G$ and $H$ is the graph with vertex set $\mathrm{V}(\mathrm{G}) \times \mathrm{V}(\mathrm{H})$ and edge set is $(u, a)(v, b) \in E(G \square H)$ if and only if $a=b$ and 


\section{G.Rajasekar and A.Venkatesan}

$u v \in E(G)$ or $u=v$ and $a b \in E(H)$ [3]. The direct product $G \times H$ of graphs $G$ and $H$ is the graph with vertex set $\mathrm{V}(\mathrm{G}) \times \mathrm{V}(\mathrm{H})$ and edge set is $(u, a)(v, b) \in E(G \times H)$ if and only if $u v \in E(G)$ and $a b \in E(H)$ [14]. The Semi-Strong Product of two graphs $\mathrm{G}$ and $\mathrm{H}$ is the graph $\mathrm{G} \bowtie H$ with vertices $\mathrm{V}(\mathrm{G} \bowtie \mathrm{H})=\mathrm{V}(\mathrm{G}) \times \mathrm{V}(\mathrm{H})$ and edges $\mathrm{E}(\mathrm{G} \bowtie \mathrm{H}$ )$=\{(\mathrm{a}, \mathrm{x})(\mathrm{b}, \mathrm{y})\}$ if and only if $(\mathrm{a}, \mathrm{b}) \in \mathrm{E}(\mathrm{G})$ and $\mathrm{x}=\mathrm{y}$ or $(\mathrm{a}, \mathrm{b}) \in \mathrm{E}(\mathrm{G})$ and $(\mathrm{x}, \mathrm{y}) \in \mathrm{E}(\mathrm{H})\}[12]$.

\section{Preliminaries}

\subsection{Location-2-domination}

Definition 2.1.1. [8] A subset $S \subseteq V$ is Location - 2 -Dominating set of $\mathrm{G}$ if $\mathrm{S}$ is 2 Dominating set of $\mathrm{G}$ and if for any two vertices $u, v \in V-S$ such that

$$
N(u) \cap S \neq N(v) \cap S .
$$

The minimum cardinality of Location-2-Dominating is denoted by $R_{2}^{D}(G)=|S|$

\subsection{Location-2-domination for simple graphs}

Theorem 2.2.1. [9] In Location-2-Domination for any graph the vertex $\{v\}$ is an pendent vertex then $\{v\} \in \mathrm{R}_{2}^{D}(G)$ only.

Theorem 2.2.2. [8] Location-2-Domination number of a Path $P_{n}$ is

$$
R_{2}^{D}\left(P_{n}\right)=\left\{\begin{array}{cc}
\frac{n-1}{2}+1, & n \text { is odd } \\
\frac{n}{2}+1 & n \text { is even }
\end{array}\right.
$$

Theorem 2.2.3. [8] Location-2-Domination for any cycle $C_{n}$, for $n \neq 4$ is

$$
R_{2}^{D}(G)=\left\{\begin{array}{cc}
\frac{n}{2} & n \text { is even } \\
\frac{n-1}{2}+1 & n \text { is odd }
\end{array}\right.
$$

\subsection{Location-2-domination for Cartesian product of graphs}

Theorem 2.3.1. [10] For any graph $G=\left(P_{n} \square S_{m}\right)$,

$$
R_{2}^{D}(\mathrm{G})=\left\{\begin{array}{cl}
R_{2}^{D}\left(\mathrm{P}_{\mathrm{n}}\right)+\frac{\mathrm{m}(\mathrm{n}-1)}{2} & n \text { is odd } \\
\frac{n}{2}(m+1) & \text { nis even }
\end{array}\right.
$$

Theorem 2.3.2. [10] Location-2-Domination for any graph $G=\left(P_{n} \square P_{m}\right)$ is 
Bounds of Location-2-Domination Number for Products of Graphs

$$
R_{2}^{D}(G)=\left\{\begin{array}{lc}
\frac{n m}{2} & n \text { is even, } m \text { is either even or odd } \\
\frac{n m-1}{2} & n \text { is odd }, \text { m is odd }
\end{array}, m \neq 2, n \neq 2\right.
$$

Theorem 2.3.3. [10] Location-2-domination for any graph $G=\left(C_{n} \square C_{m}\right)$ is

$$
R_{2}^{D}(G)=\left\{\begin{array}{cc}
\frac{n m}{2} & n \text { is even, } m \text { is either even or odd } \\
\frac{n m-1}{2} & n \text { is odd }, \text { m is odd }
\end{array}\right.
$$

\subsection{Location-2-domination for direct product of graphs}

Theorem 2.4.1. [10] For Graphs $P_{n}(n \neq 3)$ and $S_{m}, R_{2}^{D}\left(\mathrm{P}_{\mathrm{n}} \times \mathrm{S}_{\mathrm{m}}\right)=n m, n, m=1,2,3, \ldots$

Theorem 2.4.2. [10] Location -2-Domination for $P_{n}$ and $P_{m}, m \neq 3$,

$$
R_{2}^{D}\left(P_{n} \times P_{m}\right)=\left\{\begin{array}{cr}
\frac{n m}{2}+2 & n, m \text { is even } \\
\frac{n m}{2}+2 & \text { either } n \text { is odd, } m \text { is even } \\
\frac{n(m+1)}{2} & n, m \text { is even } m \text { is odd } \text { odd } n<m \\
\frac{m(n+1)}{2} & n, m \text { is odd but } n>m
\end{array}\right.
$$

Theorem 2.4.3. [10] For $n, m \geq 5$,

$R_{2}^{D}\left(C_{n} \times C_{m}\right)=\frac{n m}{2}, n, m$ is even,

$R_{2}^{D}\left(C_{n} \times C_{m}\right)=\frac{(n-1) m}{2} ; n$ is odd $m$ is even,

$R_{2}^{D}\left(C_{n} \times C_{m}\right)=\frac{n(m-1)}{2} ; n$ is even $m$ is odd,

$R_{2}^{D}\left(C_{n} \times C_{m}\right)=\frac{n(m-1)}{2} ; n, m$ is odd but $n>m$,

$R_{2}^{D}\left(C_{n} \times C_{m}\right)=\frac{m(n-1)}{2} ; n, m$ is odd but $n<m$. 


\section{Location -2-domination of products of graph}

\subsection{Location -2-domination (Cartesian product) of $\mathrm{C}_{\mathrm{n}} \square P_{m}, C_{n} \square S_{m}$}

$$
R_{2}^{D}(G)=\left\{\begin{array}{lll}
\frac{n m}{2} & n \text { is even }, \mathrm{m} \text { is either even }(\text { or }) \text { odd } & \begin{array}{l}
\text { Theorem } \\
\text { graph } P_{m} \text { and }
\end{array} \\
\frac{n m}{2}+1 & n \text { is odd } m \text { is even } & \mathrm{C}_{\mathrm{n}} \\
\frac{n m+1}{2} & n \text { is odd }, \text { m is odd } & \text { we have }
\end{array}\right.
$$

Proof: Consider path of $m$ vertices and Cycle of $n$ vertices. The Vertex set of $P_{m}$ and $C_{n}$ are $\{1,2, \ldots, m\}$ and $\{1,2, \ldots ., n\}$ respectively. Clearly $|G|=n m$ in which $2 n$ vertices are degree 3 and $(m-2) n$ vertices are degree 4 and let $S$ - Set denote Location-2Domination of G.

Case(i): Suppose $n$ is even and $m$ is either even or odd, in this situation $|V(G)|=n m$ even number of vertices. In $G$ fix any vertex from $C_{1}$ and form open path through vertices of $P_{1}$, continue the open path starts with $C_{2}$ through $P_{2}$, continue the same process till $C_{n}$ through $P_{m}$, each time the process of continuation of open path from $C_{1}$ to $C_{n}$ done only by either towards right or left direction only not alternatively. Finally the collection of vertices from $C_{1}$ to $C_{n}$ through $P_{1}$ to $P_{m}$ forms a cycle of length even with $\mathrm{nm}$ vertices. So by the Theorem: $2.2 .3,|S|=\frac{n m}{2}$, i.e. $R_{2}^{D}(G)=\frac{n m}{2}$.

Case (ii): Suppose $n$ is odd, but $m$ is even, in this situation $|V(G)|=n m$ is even, in $G$ fix any vertex from $C_{1}$ and form an open path through $P_{1}$, continue the open path starts with $C_{2}$ through $P_{2}$, continue the same process till $C_{n}$ through $P_{m}$, each time in the process of continuation open path from $C_{1}$ to $C_{n}$ done only by either towards right or left direction only not for alternatively. Finally the collection of vertices from $C_{1}$ to $C_{n}$ through $P_{1}$ to $P_{m}$ forms a path of length even with $n m$ vertices. So by the Theorem: 2.2.2 $|S|=\frac{n m}{2}+1$, i.e. $R_{2}^{D}(G)=\frac{n m}{2}+1$

Case(iii): Suppose $n$ is odd and $m$ is odd .Clearly $|G|=n m$ odd number of vertices, From $G$, let us consider $|S|=\left|S_{1}\right|+\left|S_{2}\right|$ where $\left|S_{1}\right|$ denote the Location-2-Domination for $\left\{C_{1}, C_{3}, \ldots, C_{m}\right\}$ and $\left|S_{2}\right|$ denote the Location-2-Domination for $\left\{C_{2}, C_{4}, \ldots, C_{m-1}\right\}$, but 
Bounds of Location-2-Domination Number for Products of Graphs $\left|C_{1}\right|=\left|C_{2}\right|=\ldots=\left|C_{m-1}\right|=\left|C_{m}\right|=n$. And for $S_{1}=\left\{C_{1}, C_{3}, \ldots, C_{m}\right\}$ the vertex set of $S_{1}$ are $\left\{C_{11}, C_{12}, \ldots, C_{1 n}, C_{31}, C_{32}, \ldots, C_{3 n}, \ldots, C_{m 1}, C_{m 2}, \ldots, C_{m n}\right\}$, By the Theorem: 2.2.3 Location -2Domination for Cycle of length odd is $\frac{n-1}{2}+1=\frac{n+1}{2}$. Therefore $\left|C_{i}\right|=\frac{n+1}{2}, i=1,3, \ldots, \mathrm{m}$. Clearly $S_{1}$-set contains $\frac{m+1}{2}$ times of cycle with odd length. Therefore $\left|S_{1}\right|=\left(\frac{m+1}{2}\right)\left(\frac{n+1}{2}\right)$ and $S_{2}=\left\{C_{2}, C_{4}, \ldots, C_{m-1}\right\}$ the vertex set of $S_{2}$ are $\left\{C_{21}, C_{22}, \ldots, C_{2 n}, C_{41}, C_{42}, \ldots, C_{4 n}, \ldots, C_{(m-1) 1}, C_{(m-1) 2}, \ldots, C_{(m-1) n}\right\}$, now collect the vertex from $C_{2}$ as $N\left(V-S_{1}\right)-S_{1}$ in $C_{1}$. This gives $\frac{n-1}{2}$ vertices in $C_{2}$. Continuing the same process for $\left\{C_{4}, C_{6}, \ldots, C_{m-1}\right\}$, i.e. collect the vertex for $C_{i+1}$ as $N\left(V-S_{i}\right)-S_{i}$ from $C_{i}$ for $i=1,2, \ldots, m-2$.

Clearly $S_{2}$-set contains $\frac{m-1}{2}$ times of $C_{i+1}, i=1,2, \ldots, m-2$. i.e. $\left|S_{2}\right|=\left(\frac{m-1}{2}\right)\left(\frac{n-1}{2}\right)$ $|S|=\left(\frac{m+1}{2}\right)\left(\frac{n+1}{2}\right)+\left(\frac{m-1}{2}\right)\left(\frac{n-1}{2}\right)=\frac{n m+1}{2}$. Therefore, $R_{2}^{D}(G)=|S|=\frac{n m+1}{2}$.

Theorem 3.1.2. For graphs $P_{n}$ and $S_{m}, R_{2}^{D}\left(C_{n} \square S_{m}\right)=\left\{\begin{array}{cc}\frac{n(m+1)}{2} & n \text { is even } \\ \frac{n+1}{2}+\frac{m(\mathrm{n}-1)}{2} & n \text { is odd }\end{array}\right.$

Proof: Consider the vertex set of $G$ namely $\left\{v_{i j}\right\}$ for $1 \leq i \leq n, 1 \leq j \leq m+1$. Clearly $|G|=n m$. Let $S$-set denote Location-2-Dominating set, by observing $G$, $d_{G}\left(v_{i j}\right)=m+1 \quad$ for $\quad i=1, n$. and $\quad 1 \leq j \leq m+1 \quad$ also $\quad d_{G}\left(v_{i j}\right)=4$ for $2 \leq i \leq n-1,2 \leq j \leq m+1$. i.e. $v_{i 1}, i=1,2, \ldots, n$ is adjacent with $v_{i j}, 2 \leq j \leq m+1$.

Case (i): Suppose $n$ is even and $m$ is either even or odd. Clearly $|G|=n m$ has even number of vertices, in this sense now collect $S$-set possibly by $\left\{v_{i 1}\right\}, i=1,3,5, \ldots, n-1$ and $\quad\left\{v_{i j}\right\}$ for $i=2,4, \ldots, n, 2 \leq j \leq m+1, \quad$ or $\quad\left\{v_{i 1}\right\}, i=2,4,6, \ldots, n \quad$ and $\quad\left\{v_{i j}\right\}$ for $i=1,3, \ldots, n-1,2 \leq j \leq m+1$, this gives $\frac{n}{2}$ times a single vertex and $\frac{n}{2}$ times $m$ vertices or $\left\{v_{i j}\right\}$ for $i=1,3,5, \ldots, n-1,1 \leq j \leq m+1$, this gives $\frac{n}{2}$ times $m+1$ vertices. 


\section{G.Rajasekar and A.Venkatesan}

i.e. $|S|=\frac{n}{2}+\frac{n m}{2}=\frac{n(m+1)}{2}$ therefore $R_{2}^{D}(G)=\frac{n(m+1)}{2}$.

Suppose, $\left\{v_{i 1}\right\} \in S, i=1,3,5, \ldots, n-1$ and $\left\{v_{i j}\right\} \notin S$ for $i=2,4, \ldots, n, 2 \leq j \leq m+1$ or some $\left\{v_{i j}\right\} \notin S$ for $i=2,4, \ldots, n, 2 \leq j \leq m+1$. Clearly this contradicts the definition of Location-2-Domination or minimum cardinality of $S$ - set or $\left\{v_{i 1}\right\} \notin S, i=1,3,5, \ldots, n-1$ and $\left\{v_{i j}\right\} \in S$ for $i=2,4, \ldots, n .2 \leq j \leq m+1, \quad$ in this situation $\left\{v_{i j}\right\}, i=1,3,5, \ldots, n-1$, $1 \leq j \leq m+1$ needs additional vertex, clearly it also contradicts the minimum cardinality of $S-$ set.

Case (ii): Suppose $n$ is odd, $m$ is either even or odd. Clearly $|V(G)|=n m$ gives even number of vertices, in this sense now collect $S$ - set possibly by $\left\{v_{i 1}\right\}, i=1,3,5, \ldots, n$ and $\left\{v_{i j}\right\}$ for $i=2,4, \ldots, n-1.2 \leq j \leq m+1$, this gives $\frac{n+1}{2}$ times a single vertex and $\frac{n-1}{2}$ times $m+1$ vertices.

That is, $|S|=\frac{n+1}{2}+\frac{(n-1) m}{2}$ and therefore $R_{2}^{D}(G)=\frac{n+1}{2}+\frac{m(n-1)}{2}$.

Suppose, $\left\{v_{i 1}\right\} \in S, i=2,4, \ldots, n-1$ and $\left\{v_{i j}\right\} \in S$ for $i=1,3, \ldots, n, 2 \leq j \leq m+1$ this gives $|S|=\frac{n-1}{2}+\frac{(n+1) m}{2}$ contradicts minimum cardinality of $S$-set or some $\left\{v_{i j}\right\} \notin S$ for $i=2,4, \ldots, n, 2 \leq j \leq m+1$, clearly it contradicts the definition of Location-2-Domination.

\subsection{Location-2-domination (Direct product) of $P_{n} \times W_{m}, P_{n} \times C_{m}$}

Theorem 3.2.1. For any Graphs $P_{n}, n \neq 2$ and $W_{m}, m \neq 5$ we have

$$
R_{2}^{D}(G)=\left\{\begin{array}{cl}
\frac{n m}{2} & n \text { is even } \\
\frac{m(n+1)}{2} & \text { nis odd }
\end{array}\right.
$$

Proof: Label the vertices of $G$ as $\left\{v_{i j}\right\}, 1 \leq i \leq n, 1 \leq j \leq m$, clearly $|G|=n m$ from $G$ $d_{G}\left(v_{11}\right)=d_{G}\left(v_{n 1}\right)=m-1, \quad d_{G}\left(v_{1 j}\right)=d_{G}\left(v_{n j}\right)=3,2 \leq \mathrm{j} \leq \mathrm{m}$ and $\quad d_{G}\left(v_{i j}\right)=6$, $2 \leq i \leq n-1, \quad 2 \leq j \leq m$. Now labels of $G$ are partitioned into $n$ different sets namely $U_{i}, 1 \leq \mathrm{i} \leq \mathrm{n}$ are $\left\{v_{i j}\right\}, 1 \leq i \leq n, 1 \leq j \leq m$ respectively. But there is no adjacency from $v_{i j}$ to $v_{j i}$ for $1 \leq i \leq n, 1 \leq j \leq m$. Clearly $u_{i}$ is adjacent to $u_{i-1}$ and $u_{i+1}$ for $i=1,2, \ldots, n$. 
Bounds of Location-2-Domination Number for Products of Graphs

Case (i): Suppose $n$ is even, then the collection of the sets $U_{i}, i=1,3, \ldots, n-1$ or $i=2,4, \ldots, n$ will have $\frac{n}{2}$ times $m$ vertices i.e. $|S|=\frac{n m}{2}$ therefore $R_{2}^{D}(G)=\frac{n m}{2}$.

Case (ii): Suppose $n$ is odd by based on Theorem 2.2.2, the collection of the $\operatorname{sets} U_{i}$, $i=2,4, \ldots, n-1$ will $\quad$ have $\frac{n-1}{2}$ times $\quad m$ vertices $\quad$ i.e. $|S|=\frac{(n-1) m}{2} \quad$ therefore $R_{2}^{D}(G)=\frac{(n-1) m}{2}$. Suppose if we collect the sets $U_{i}, i=1,3, \ldots, n$ this contradicts the minimum cardinality.

Result 3.2.1. $R_{2}^{D}\left(P_{2} \times W_{m}\right)=m$.

Result 3.2.2. $R_{2}^{D}\left(P_{n} \times W_{5}\right)=\left\{\begin{array}{cc}3 n, & n \text { is even } \\ 5\left(\frac{n+1}{2}\right)+\frac{n-1}{2}, & n \text { is odd }\end{array}\right.$

Theorem 3.2.2. For Graphs $C_{n}$ and $S_{m}, R_{2}^{D}\left(C_{n} \times S_{m}\right)=n m n, m=1,2,3, \ldots$

Proof: The vertex set of $G$ are $\left\{v_{i j}\right\}, 1 \leq i \leq n, 1 \leq j \leq m+1$. Let $S-$ set denote Location-2-Dominating set of $G$. Clearly by observation of $G d_{G}\left(v_{i 1}\right)=2 m, 1 \leq i \leq n$ and $d_{G}\left(v_{i j}\right)=2,1 \leq i \leq n, 2 \leq j \leq m+1$. Now collect the $S-$ set possibly by either $v_{i j}, 1 \leq i \leq n, 2 \leq j \leq m+1$ or $\quad v_{i 1}, 1 \leq i \leq n$ and $\quad v_{i j}, 1 \leq i \leq n, 3 \leq j \leq m+1 \quad$ i.e. leaving anyone of the same base vertex of $i=1,2, \ldots$ or $j=1,2, \ldots$ clearly this $n$ times $m$ vertices. i.e. $|S|=n m$. Suppose $v_{i 1} \in S, i=1,2,3, \ldots, n$ and $v_{i j} \notin S, 1 \leq i \leq n, 2 \leq j \leq m+1$ then this contradicts the definition of Location-2-Domination. Therefore $R_{D}^{2}(G)=|S|=n m$.

3.3 Location-2-domination (semi-strong product) of $P_{n} \bowtie P_{m}, C_{n} \bowtie P_{m}, C_{n} \bowtie C_{m}$ Theorem 3.3.1. For any graphs $P_{n}, n \neq 3$ and $P_{m}, m \neq 2, \mathrm{G}=\mathrm{P}_{\mathrm{n}} \bowtie \mathrm{P}_{\mathrm{m}}$ is

$$
R_{2}^{D}(G)=\left\{\begin{array}{cc}
\frac{n m}{2}, & n \text { is even, } m \text { is even or odd } \\
\frac{n m}{2}+2, & n \text { is odd, } m \text { is even } \\
\frac{n(m+1)}{2}, & n, \text { is odd } n<m \\
\frac{m(n+1)}{2}, & n, m \text { is odd } m<n \\
\frac{n(m+1)}{2}, &
\end{array}\right.
$$




\section{G.Rajasekar and A.Venkatesan}

Proof: Label the vertices of $G$ as $\left\{v_{i j}\right\}, 1 \leq i \leq n$ and $1 \leq j \leq m$. Let $S-$ set be the Location-2-Domination set of $G$, then clearly $d_{G}\left(v_{i j}\right) \geq 2, i=1,2, . ., n j=1,2, \ldots, m$.

Case (i): Suppose $n$ is even and $m$ is even or odd, now let us collect the S-set possibly by $\left\{v_{i j}\right\} i=1,3, \ldots, n-1, j=1,2, \ldots, m$ or $\left\{v_{i j}\right\} i=2,4, \ldots, \mathrm{n}, j=1,2,3, \ldots, m$ this gives $\frac{n m}{2}$ vertices i.e. $|S|=\frac{n m}{2} R_{2}^{D}(G)=\frac{n m}{2}$.

Case (ii): Suppose $n$ is odd, $m$ is even in this sense let us collect the $S$-set possibly by $\left\{v_{i j}\right\} i=1,2,3, \ldots, n, j=1,3, \ldots, m-1$ and $v_{1 m}, v_{n m}$ and this gives $\frac{n m}{2}+2$ vertices that is $|S|=\frac{n m}{2}+2$ therefore $R_{2}^{D}(G)=\frac{n m}{2}+2$. Suppose the vertices $v_{1 m}, v_{n m}$ does not belong to $S$-set or $\left\{v_{i j}\right\} i=1,3, \ldots, n-1, j=1,2,3, \ldots, m$ then this is a contradiction to minimum cardinality.

Case (iii): Suppose $n, m$ is odd but $n<m$, in this case let us collect the $S$-set possibly by $\left\{v_{i j}\right\} i=1,2,3, \ldots, n, j=1,3, \ldots, m-1$ and this gives $n$ times $\frac{m+1}{2}$ vertices that is $|S|=\frac{n(m+1)}{2}$ therefore $R_{2}^{D}(G)=\frac{n(m+1)}{2}$. Then the collection $\left\{v_{i j}\right\} i=1,3, \ldots, n-1$, $j=1,2,3, \ldots, m$ is not a minimum cardinality set.

Case (iv): Similar to the case (iii).

Case (v): suppose $n, m$ is odd but $n=m$ in this case let us collect the $S$-set possibly by $\left\{v_{i j}\right\} i=1,2,3, \ldots, n, j=1,3, \ldots, m \quad$ or $\quad\left\{v_{i j}\right\} i=1,3, \ldots, n, j=1,2,3, \ldots, m$ then this gives $\frac{n(m+1)}{2}$ vertices that is $|S|=\frac{n(m+1)}{2}$ and hence $R_{2}^{D}(G)=\frac{n(m+1)}{2}$.

Result 3.3.1. $R_{2}^{D}\left(P_{n} \times P_{2}\right)=n$

Result 3.3.2. $R_{2}^{D}\left(P_{3} \times P_{m}\right)=2 m$

Observation 3.3.1. The semi-strong product of $C_{n} \times P_{m}$ is not equal to $P_{n} \times C_{m}$

Theorem 3.3.2. For any graphs $C_{n}, \mathrm{n}>5$ and $P_{m}, \mathrm{~m} \neq 2, \mathrm{G}=\mathrm{C}_{\mathrm{n}} \bowtie \mathrm{P}_{\mathrm{m}}$ 
Bounds of Location-2-Domination Number for Products of Graphs

$$
R_{2}^{D}(\mathrm{G})=\left\{\begin{array}{rr}
2\left(\frac{m}{2}+1\right)+\frac{m}{2}+m\left(\frac{n-4}{2}\right) & n, m \text { is even } \\
(m+1)+m\left(\frac{n-3}{2}\right) & n, \text { mis odd } \\
m\left(\frac{n-3}{2}\right)+2(m+2) & n \text { is odd } m \text { is even } \\
3\left(\frac{m+1}{2}\right)+m\left(\frac{n-4}{2}\right) & n \text { is even } m \text { is odd }
\end{array}\right.
$$

Proof: $|V(G)|=n m=\left\{v_{i j}\right\}, i=1,2, \ldots, n, j=1,2, \ldots, m$.Clearly $d_{G}\left(v_{i j}\right) \geq 2$ for $i=1,2, . ., n$, $j=1,2, \ldots, m$

Case (i): Suppose $n, m$ is even, in this case cardinality of $S$ - set contains the vertices are $v_{i j}, i=1, n . j=1,3, \ldots, m-1, m$ and $\quad v_{(n-1) j}, j=1,3, \ldots, m-1 \quad$ also $\quad v_{i j}$ for $i=3,5, \ldots, n-3$. $j=1,2, \ldots, m$. Clearly this gives 2 times $\left(\frac{m}{2}+1\right)$ vertices and $\frac{m}{2}$ times a single vertex. Also $\frac{n-4}{2}$ times $m$ vertices. That is $|S|=2\left(\frac{m}{2}+1\right)+\frac{m}{2}+m\left(\frac{n-4}{2}\right)$ and therefore $R_{2}^{D}(G)=2\left(\frac{m}{2}+1\right)+\frac{m}{2}+m\left(\frac{n-4}{2}\right)$.

Case (ii): Suppose $n, m$ is odd, now the $S$-set contains the vertices $v_{i j}, i=1, n . j=1,3, \ldots, m$ and also $v_{i j}$ for $i=3,5, \ldots, n-2, j=1,2, \ldots, m$. Then this gives 2 times $\left(\frac{m+1}{2}\right)$ vertices and $\frac{n-3}{2}$ times $m$ vertices. That is $|S|=(m+1)+m\left(\frac{n-3}{2}\right)$ and therefore $R_{2}^{D}(G)=(m+1)+m\left(\frac{n-3}{2}\right)$

Case (iii): Proof is similar to Case (i) and hence $R_{2}^{D}(G)=(m+2)+m\left(\frac{n-3}{2}\right)$

Case (iv): Suppose $n$ is even, $m$ is odd, now the $S$-set contains the vertices $v_{i j}, i=1, n-1, n ; j=1,3, \ldots, m$ and also $v_{i j}$ for $i=3,5, \ldots, n-3 ; j=1,2, \ldots, m$. Then this gives 3 times $\left(\frac{m+1}{2}\right)$ vertices and $\frac{n-4}{2}$ times $m$ vertices. That is $|S|=3(m+1)+m\left(\frac{n-4}{2}\right)$ therefore $R_{2}^{D}(G)=3(m+1)+m\left(\frac{n-4}{2}\right)$ 
Result 3.3.3. $R_{2}^{D}\left(C_{2} \times C_{m}\right)=m, m \neq 2,3$

Result 3.3.4. $R_{2}^{D}\left(C_{3} \times C_{m}\right)=2(m-1)$

Result 3.3.5. $R_{2}^{D}\left(C_{4} \times C_{m}\right)=2 m$

Theorem 3.3.3. For Graphs $C_{n}, n \neq 2,3,4$. and $C_{m}, \mathrm{G}=\mathrm{C}_{\mathrm{n}} \bowtie \mathrm{C}_{\mathrm{m}}$ is

$$
R_{2}^{D}(\mathrm{G})=\left\{\begin{array}{rr}
\frac{n m}{2} & n, m \text { is even } \\
n\left(\frac{m-1}{2}\right) & n \text { is even or odd, } m \text { is odd } \\
m\left(\frac{n-1}{2}\right) & n \text { is odd }, \text { m is even }
\end{array}\right.
$$

Proof: Let the vertices of $G$ be $\left\{v_{i j}\right\}, i=1,2, \ldots, n, j=1,2, \ldots, m$.Let $S$-denotes Location2-Dominating set.

Case (i): Proof is followed by Theorem 3.5 Case (i)

Case (ii): Suppose $n$ is odd, $m$ is odd and let us collect the $S$-set possibly by $S$ $\left\{v_{i j}\right\} i=1,3, \ldots, n-2, j=1,2,3, \ldots, m$. Clearly this gives $\frac{m-1}{2}$ times $n$ vertices,that is $|S|=n\left(\frac{m-1}{2}\right)$ and therefore $R_{2}^{D}(G)=n\left(\frac{m-1}{2}\right)$.

Case (iii): suppose $n$ is odd, $m$ is even and let us collect the $S$-set possibly by $\left\{v_{i j}\right\} i=1,2,3, \ldots, n, j=1,3, \ldots, m-1$. Clearly this gives $\frac{n-1}{2}$ times $m$ vertices that is $|S|=m\left(\frac{n-1}{2}\right)$ and hence $R_{2}^{D}(G)=m\left(\frac{n-1}{2}\right)$. Suppose if anyone the vertex collection as $\left\{v_{i j}\right\} i=1,3, \ldots, n-2, n-1, j=1,2,3, \ldots, m$ then this is once againa contradiction to minimum cardinality.

Acknowledgement. The authors thank the anonymous referees for their helpful suggestions.

\section{REFERENCES}

1. M. El-Zahar and C.M. Pareek, Domination number of products of graphs, Ars Combine., 31 (1991) 223-227. 
Bounds of Location-2-Domination Number for Products of Graphs

2. M. El-Zahar, S. Khamis and Kh. Nazzal, On the domination number of the Cartesian product of the cycle of length $\mathrm{n}$ and any graph, Discrete App. Math., 155 (2007) 515522.

3. R.J. Faudree and R.H. Schelp, The domination number for the product of graphs, Congr. Numer., 79 (1990) 29-33.

4. S.Gravier and M.Mollard, On Domination numbers of Cartesian product of paths, Discrete App. Math., 80 (1997) 247-250.

5. F.Harary and T.W.Haynes, Double domination in graphs, Ars Combin., 55 (2000) 201-213.

6. F.Haray, Graph Theory, Addsion Wesley, Reading, MA (1969).

7. T.W.Haynes, S.T.Hedetniemi and P.J.Salter, Fundamental of domination in Graphs, Marcel Decker ,Inc., New York (1997).

8. G.Rajasekar, and A.Venkatesan, Location-2-domination for simple graphs, Global Journal of Pure and Applied Mathematics, 13(9) (2017) 5049-5057.

9. G.Rajasekar and A.Venkatesan, Location-2-domination for special kinds of simple graphs, International Journal of Pure and Applied Mathematics, 117(5) (2017)13-20.

10. G.Rajasekar and A.Venkatesan, Location-2-domination for product of graphs, communicated.

11. P.J.Salter, Dominating and refrence sets in graphs, J. Math. Phy. Sci, 22 (1988) 445-455.

12. S.R.Cheney, Domination Numbers of Semi-strong Products of Graphs, Thesis and Dissertations, Virginia Commonwealth University.

13. S.Klaviar and N.Seifter, Dominating Cartesian products of cycles, Discrete Applied Mathematics, 59 (1995) 129-136.

14. T.Sitthiwirattham, Domination on Kronecker product of $P_{n}$, Applied Mathematical Sciences, 6 (2012) 4345 - 4352. 